\title{
BMES Editorial
}

The Biomedical Engineering Society (BMES) Annual Meeting in Atlanta, Georgia in October of 2012 was again highly successful, with an excellent set of contributions in the area of Cellular and Molecular Bioengineering.

To recognize excellence in the area of Cellular and Molecular Bioengineering as presented at the BMES meeting, we have organized a Special Issue of $C M B E$. We thank the BMES Cellular and Molecular Bioengineering Track reviewers, who evaluated the submitted abstracts and provided us with a scoring basis for selecting the most outstanding work. Also, we thank Cellular and Molecular Bioengineering Track Co-Chairs Scott Diamond (University of Pennsylvania) and Wilbur Lam (Emory University), for their support of the Special Issue.

Contained in this issue are four of the most outstanding cellular and molecular bioengineering papers that were presented at the 2012 BMES meeting:

John Huynh, Francois Bordeleau, Casey KraningRush, and Cynthia Reinhart-King describe how circular dorsal ruffles are induced by the combined chemical effects of PDGF and mechanical effects of substrate stiffness in vascular smooth muscle cells. These cytoskeletal features may play an important role in the migration of smooth muscle cells into the intimal layer, where hyperproliferation can promote atherosclerotic plaque formation.

Saheli Sarkar, Christine Payne, and Melissa Kemp examined the nuclear factor erythroid 2-related factor-2 (Nrf2) transcription factor that activates genes in response to oxidative stress. They found that the Nrf2 is level is strongly dependent on the media conditions used, without observable effects on Keap1, the primary inhibitor of Nrf2. They suggest that Nrf2 may actually regulate itself, which will be important to integrate into models for $\mathrm{Nrf2}$ response to oxidative stress.

Nandini Shekhar, Tanmay Lele, and co-workers investigated the mechanisms by which microtubules bend in fibroblast cells. They found that bending was driven largely by dynein motors, rather than myosin motors. Because microtubules play a vital role in cell polarization and cytoplasmic organization, the findings suggest that the organization of the microtubule array in interphase cells will be controlled by microtubule-dependent motors rather than the actin cortex.

Yue Geng, Michael King, and co-workers reported on the underglycosylated form of the glycoprotein MUC1, and its role in breast cancer cell adhesion. They found cell line sensitivity to different selectins in rolling assays, an effect that they also investigated through molecular dynamics simulations. Using a combination of adhesion-mediating molecules, they found good capture efficiency from whole blood, which suggests novel approaches to the capture of highly metastatic circulating tumor cells.We hope that you will enjoy reading these exciting Outstanding Papers from the 2012 BMES Meeting.

DAvid J. OdDE

Department of Biomedical Engineering University of Minnesota, MN, USA

Electronic mail: oddex002@umn.edu

FRANK YIN

Department of Biomedical Engineering

Washington University, St. Louis, MO, USA 